Journal of Computer Science 7 (6): 836-843, 2011

ISSN 1549-3636

(C) 2011 Science Publications

\title{
Weighted Centroid Modified Simplex and Linear Constrained Response Surface Optimization Methods for the Xbar-R Chart Variable Determination
}

\author{
Pongchanun Luangpaiboon \\ Department of Industrial Engineering, Faculty of Engineering, \\ Industrial Statistics and Operational Research Unit, \\ Thammasat University, Pathumthani, 12120, Thailand
}

\begin{abstract}
Problem statement: Although economic Xbar-R chart designs do guarantee the minimal operating cost, they typically have poor levels of statistical performance measures. The obvious limitation of the economic design is that the Type I error rate seems to be very high for many situations and will cause a large number of false alarms. This situation leads to an investigation of appropriate levels of control chart variables which consist of a sample size, an interval between samples or sampling frequency and the control chart limits. Approach: Evolutionary operations via the weighed centroid modified simplex, WCMSM and linear constrained response surface optimization, LCRSOM, methods are applied to optimize the Xbar-R chart variables in the analytical model called as the operating cost function. WCMSM allows the simplex to converge more rapidly towards an optimum via the weighted centroid of the hyperface by expansion and multiple ways of simplex contraction along the line of conventional reflection in order to speed up the convergence. LCRSOM is a combination of the principles of experimental designs, least squares and related mathematical programming models to approach the optimum. Results: The computational results for economic Xbar-R charts via both methods reveal that the process quality level affects the total cost more than relevant errors from selecting and inspecting samples including identifying assignable causes. However, total costs obtained from the LCRSOM seem to be better for all process scenarios. Conclusions: On Xbar-R charts, if a process is at the high quality level the total cost is better than a process with a low quality level without a consideration of errors from selecting/inspecting samples and identifying assignable causes.
\end{abstract}

Key words: Economic design, Xbar-R chart, type I error, Evolutionary Operation (EVOP), response surface, weighted centroid modified simplex method, linear constrained response surface, optimization method

\section{INTRODUCTION}

Control charts are widely used to establish and maintain statistical control of a process. They have been claimed to be effective devices for determining process variables (Abu-Shawiesh, 2008). An application of control charts requires a proper level of sample size, an interval between samples or sampling frequency and the control limits for a chart. Optimal variable setting is called the economic design of the control chart, first introduced by Girshick and Rubin.

In the economic design of control charts it is assumed that the process is characterized by an incontrol state with a central tendency of a process, $\mu$ and that a single assignable cause of shift magnitude to be detected in the process mean, $\delta$, which randomly occurs, results in a shift in the mean from $\mu$ to either $\mu+\delta \sigma$ or $\mu-\delta \sigma$. The process is monitored by an Xbar chart with the centerline of $\mu$ and upper and lower control limits of $\mu \pm k \sigma / \sqrt{n}$, where $\sigma$ is a process standard deviation, $\mathrm{k}$ is a control limit coefficient and $\mathrm{n}$ is a sample size (Carolan et al., 2010). Samples are to be taken at intervals of $h$ hours. The decision variables of $\mathrm{n}, \mathrm{h}$ and $\mathrm{k}$ are determined to achieve the minimal expected operating cost per unit time (Baud-Lavigne et al., 2010).

On situations with higher Type I error rate and a large number of false alarms, designers of economic control charts are not able to determine a priori what the control chart statistical properties will actually be. In addition, they cannot determine how sensitive the cost is to the improvement of these properties. A statistically designed control chart is a structured method in which the Type I error probability and power are usually fixed at the desired levels. 
The economic statistical design combines the benefits of both pure statistical and economic designs while minimizing their weaknesses, or a Type I error rate (Yang et al., 2010; Yang and Rahim, 2009). An objective is to determine the optimal levels of variable control chart variables via analytical models of the total operating cost or price function and statistical parameters properties.

\section{MATERIALS AND METHODS}

Economic Design of the Xbar-R Chart: Variation occurs in all industrial processes. Common cause variation is a natural part of the process. Some special causes are other type of variations. They come from outside the system and cause recognizable patterns, shifts, or trends in the data. The control charts show if special causes are influencing the process considered. Variables control charts provide statistics from measurement data for subgroup data. Variables control charts for individuals, time-weighted charts and multivariate charts also plot measurement data. There are various assumptions underlying the theory used in formulating the variable control charts when applied to the industrial processes (Matouq et al., 2007).

With the preset level of a width factor of $k_{1}$, the sample mean or Xbar chart is maintained to detect the occurrence of assignable cause(s) between upper and lower control limits of $\mu_{0}+\mathrm{k}_{1} \frac{\sigma}{\sqrt{\mathrm{n}}}$ and $\mu_{0}-\mathrm{k}_{1} \frac{\sigma}{\sqrt{\mathrm{n}}}$, respectively. With the preset level of a width factor of $\mathrm{k}_{2}$, the sample range or R chart is maintained to detect the occurrence of assignable cause(s) between the lower and upper control limit of 0 and $k_{2} \sigma_{0}$, respectively. The distribution of occurrences of assignable causes is Poisson distribution with the mean of $\lambda$ units per hour. The occurrence times for assignable causes are independently exponentially distributed with the meantime of $1 / \lambda$.

When there is an evidence of out of control, the process will be kept running. A Cycle Time (CT) is a time period of a process changing from in - out of control stage. An average time $(\tau)$ between the $j$-th sample and the $\mathrm{j}+1$-th sample, taken just prior to the occurrence of the assignable cause and the occurrence, can be defined as follows Eq. 1:

$$
\mathrm{CT}=\frac{1}{\lambda}+\frac{\mathrm{h}}{(1-\beta)}-\tau+\mathrm{g} * \mathrm{n}+\mathrm{D}
$$

Where:

$$
\tau=\frac{\int_{j h}^{(j+1) h} e^{-\lambda t} \lambda(t-j h) d t}{\int_{j h}^{(j+1) h} e^{-\lambda t} \lambda d t}
$$

Or:

$$
\frac{1-(1+\lambda \mathrm{h}) \mathrm{e}^{-\lambda \mathrm{h}}}{\lambda\left(1-\mathrm{e}^{-\lambda \mathrm{h}}\right)}
$$

The analytical models of the expected total cost per unit time (E) and statistical properties of Xbar-R chart are given below. The total cost consist of the cost or price per hour of false alarms before process parameters go out of control $\left(\mathrm{E}_{1}\right)$ with A-expected number of false alarms when a process is in control, the cost of out of control condition $\left(\mathrm{E}_{3}\right)$, the cost of identifying assignable cause $\left(E_{2}\right)$ and finally the cost of sampling and inspection $\left(\mathrm{E}_{4}\right)$ Eq. 2-8:

$\mathrm{E}=\mathrm{E}_{1}+\mathrm{E}_{2}+\mathrm{E}_{3}+\mathrm{E}_{4}$

$\mathrm{E}_{1}=\frac{\mathrm{A} * \mathrm{~F}}{\mathrm{CT}}$

$E_{2}=\frac{\left[\frac{h}{(1-\beta)}-\tau+g * n+D\right] M}{C T}$

$\mathrm{E}_{3}=\frac{\mathrm{W}}{\mathrm{CT}}$

$\mathrm{E}_{4}=\frac{\mathrm{b}}{\mathrm{h}}+\frac{\mathrm{c} * \mathrm{n}}{\mathrm{h}}$

Where:

$A=\alpha\left[\sum_{L=0}^{\infty} \int_{L h}^{(L+1) h} L \lambda e^{-\lambda t} d t\right]=\alpha\left[\frac{e^{-\lambda h}}{\left(1-e^{-\lambda h}\right)}\right]$

Where:

$\mathrm{F}=$ Average cost per occasion of looking for an assignable cause when none exists

$\beta=$ Probability of Type II error

$\mathrm{g}=$ Average time between taking a sample

$\mathrm{D}=$ Average time of finding the out of control condition after being detected

$\mathrm{M}=$ Average cost per hour of operation due to the out of control (from an assignable cause) 
$\mathrm{W}=$ Average cost of determining the out of control condition when it happens,

$\mathrm{b}=$ Fixed cost of sampling and testing

$\mathrm{c}=$ Variable cost of sampling and testing

$\lambda=$ Failures occurrence rate per hour:

$\beta=1-\left(\mathrm{P}_{\overline{\mathrm{X}}}+\mathrm{P}_{\mathrm{R}}-\mathrm{P}_{\overline{\mathrm{X}}} \mathrm{P}_{\mathrm{R}}\right)$

Where:

$$
\begin{aligned}
& \mathrm{P}_{\overline{\mathrm{X}}}=1-\left(\phi\left[\frac{\mathrm{k}_{1}-\delta \sqrt{\mathrm{n}}}{\gamma}\right]-\phi\left[\frac{-\mathrm{k}_{1}-\delta \sqrt{\mathrm{n}}}{\gamma}\right]\right) \\
& \mathrm{P}_{\mathrm{R}}=1-\phi\left[\frac{\frac{\mathrm{k}_{2}}{\gamma} \mathrm{d}_{2}}{\mathrm{~d}_{3}}\right]
\end{aligned}
$$

Where:

$\gamma=$ Shift detected magnitude in the dispersion

$\mathrm{P}=$ Power of a control chart or the probability that the control charts indicate false alarm

$\mathrm{P}_{\overline{\mathrm{X}}}$ and $\mathrm{P}_{\mathrm{R}}=$ Power for Xbar and $\mathrm{R}$ charts:

$\alpha=\alpha_{\overline{\mathrm{X}}}+\alpha_{\mathrm{R}}-\alpha_{\overline{\mathrm{X}}} \alpha_{\mathrm{R}}$

Where:

$$
\begin{aligned}
& \alpha_{\overline{\mathrm{x}}} 2\left(1-\phi\left(\mathrm{k}_{1}\right)\right) \\
& \alpha_{\mathrm{R}}=1-\phi\left[\frac{\mathrm{k}_{2}-\mathrm{d}_{2}}{\mathrm{~d}_{3}}\right]
\end{aligned}
$$

Where:

$\alpha_{\overline{\mathrm{x}}}$ and $\alpha_{R}=$ Probability of Type I error for Xbar and $\mathrm{R}$ charts

$\mathrm{d}_{2}$ and $\mathrm{d}_{3}=$ Control chart constants for mean and standard deviation

The objective of this research is then to minimize the expected total operating cost per unit time. A simulation study is based on the function of process variables of economic design of Xbar-R control chart models. The weighted centroid modified simplex and linear constrained response surface optimization methods are applied to find the values of the process variables which give the lowest yield. Conclusions are drawn and practical recommendations are made.

Linear constrained response surface optimization method (LCRSOM): Evolutionary operations aim to lead the experimenter rapidly and efficiently along a path of improvement toward the general vicinity of the optimum. If the true optimum is a point of minimal response, then we may think of "descending into a valley". A response surface describes how the yield of a process varies with changes in $\mathrm{m}$ independent variables. Estimation of such surfaces and hence identification of near optimal settings for predictor variables is an important practical issue with interesting theoretical aspects. Many systematic methods for making an efficient empirical investigation of such surfaces have been proposed in the last fifty years.

The procedure of the LCRSOM is that a hyperplane is fitted to the results from the initial $2^{\mathrm{k}}$ factorial designs. The data from these design points are analysed. If there is an evidence of main effect(s), at some chosen level of statistical significance and no evidence of curvature, at the same level of significance, the direction of steepest descent on the hyperplane $(\hat{y})$ is then determined by using principles of least squares and experimental designs. In order to achieve the linear mathematical model of the LCRSOM is then formulated with a consideration of the feasible ranges in terms of Lower (LB) and Upper (UB) bounds of all influential variables (x), namely:

Optimize $\hat{y}$

Subject to:

$\mathrm{LB} \leq \mathrm{x} \leq \mathrm{UB}$

The next run is carried out at a point, which has some fixed distance in this direction and further runs are carried out by continuing in this direction until no further decrease in yield is noted. When the response first increases and no improvement of two more verified yields, another $2^{\mathrm{k}}$ factorial design will be carried out, centered on the preceding design point. A new direction of steepest descent is estimated from this latest experiment (Ismail et al., 2009). Provided at least one of the coefficients of the hyperplane is statistically significantly different from zero, the search continues in this new direction (Fig. 1). Once the first order model is determined to be inadequate, the area of optimum is identified via a second order model or a finishing strategy.

Practically, a first-order approximation will be used as a proper local search in a small region close to the initial operating conditions and far from where the process determines a curvature effect. It therefore makes sense for fitting a simple first-order or linear polynomial model from available design points. 
Procedure of the LCRSOM ()

While (termination criterion not satisfied) - (line 1)

Schedule activities (when regression verification criteria not satisfy)

Determine significant first order model from the factorial design points;

Determine the mathematical models of LCRSOM; Schedule activities

Move along the estimated variable levels with a step length $(\Delta)$;

Compute imbalance values;

If new imbalance design point is smaller than the preceding then move ahead with another $\Delta$;

Else

Calculate two more imbalance design points to verify the descending trend;

If One of which imbalance design point turn out to be smaller than preceding design point's imbalance then

Use the smallest imbalance to continually move along the same path

else

Use closest preceding design point as a centre for new $2^{\mathrm{k}}$

design;

End if;

End if;

End schedule activities;

End schedule activities:

End while;

End procedure;

Fig. 1: Pseudo code of the LCRSOM

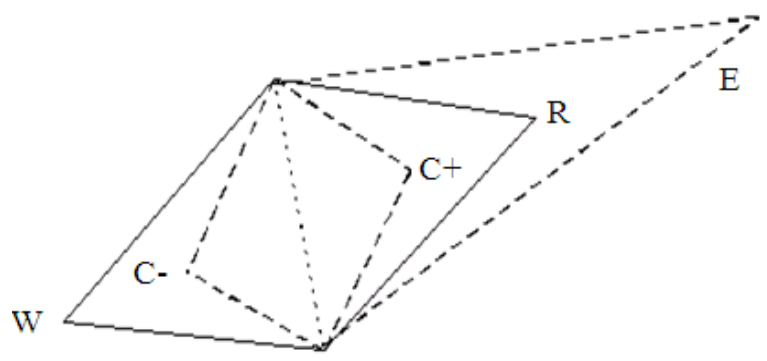

Fig. 2: Different simplex moves from the rejected trial condition (W). $\mathrm{R}=$ reflection, $\mathrm{E}=$ expansion, $\mathrm{C}+$ $=$ positive contraction and $\mathrm{C}-=$ negative contraction

This mathematical model in forms of the search direction or path of steepest ascent (or descent) is measured to locate the new design points with a preset step length until there is no further improvement in the response (Kamoun et al., 2009). When there is a lack of fit or significant pure quadratic curvature effect, it can be implied that influential variable levels are close to where the maximum or minimum occurs. A secondorder polynomial model or a canonical analysis of the surface can be used as a local approximation of the response in a small region where the optimal levels of influential variables exist.

Weighted Centroid Modified Simplex Method (WCMSM): A simplex is an m-dimensional polyhedron with $\mathrm{m}+1$ vertices, where $\mathrm{m}$ is the number of decision variables for optimisation or the dimension of the search space. This sequential optimum search is based on moving away from the experiment with the worst result in a simplex consisting of $\mathrm{m}+1$ experiments. The objective of the sequential simplex method is to drive the simplex toward the region of the factor space which is of optimal response. A new symmetrical simplex consists of one new point and $\mathrm{m}$ design points from the previous simplex or discarding the worst point and replacing it with a new point. Repetition of simplex reflection and response measurement forms the basis for the simplex algorithm (Farshi et al., 2011; Zhao et al., 2009).

Many modifications to the original simplex algorithm have been developed. Nelder and Mead modified a basic simplex method to allow various procedures to adapt to the response surface much more readily than the original. This method is referred to modified simplex method. MSM allows the simplex to converge more rapidly towards an optimum by expansion and multiple ways of contraction along the line of conventional reflection in order to speed up the convergence. When the response is more preferable than the responses of the previous vertices, expansion with a preset expansion coefficient is applied, to stretch the move beyond the simple reflection (Fig. 2).

In some cases when the response is more desirable than the worst one, but still worse than all the remaining responses, contraction with a preset contraction coefficient is applied to make the move shorten in comparison to the reflection. Moreover, massive contractions are applied when the new response gets worse than any of the previous ones. In this case the size of the simplex is reduced by contracting each of its edges to one half of its previous length toward the vertex producing the best response. A new simplex is thus generated with $m$ new measurements and the sequential optimisation procedures are repeated (Luangpaiboon, 2010).

The algorithmic details of the WCMSM are similar to the original MSM as above (Fig. 3). However, the subsequent vertex is projected with a preset reflection coefficient to the weighted centroid of the hyperface (WC) instead of the common centroid. 
J. Computer Sci., 7 (6): 836-843, 2011

Procedure of the WCMSM Meta-heuristic()

While (termination criterion not satisfied) - (line 1)

Schedule activities

Reflection of least yield $\mathrm{W}$ is processed;

Compute WC, R and $\mathrm{f}(\mathrm{R})$;

Compare response function;

If $f(R)$ is highest then

Extension E will be processed;

Else

If $R$ and $f(R)$ continue to be the least then Reflect backward to prior point; Recalculate $\mathrm{W}$ and $\mathrm{f}(\mathrm{W})$;

or

Contraction $\mathrm{C}$ or shrinking $\mathrm{S}$ will be processed;

Recalculate $\mathrm{f}(\mathrm{C})$ or $\mathrm{f}(\mathrm{S})$;

Else

Go to line 3;

End if;

End if;

End schedule activities;

End while;

End procedure;

Fig. 3: Pseudo code of the WCMSM

The new vertex is formed by the remaining simplex design points (i) with an opposite direction from the worst vertex $(\mathrm{W})$, where:

$\mathrm{WC}=\frac{\sum|\mathrm{f}(\mathrm{i})-\mathrm{f}(\mathrm{W})| \mathrm{i}}{\sum|\mathrm{f}(\mathrm{i})-\mathrm{f}(\mathrm{W})|}$

\section{RESULTS}

To illustrate the effects of economic statistical designs of the Xbar- $\mathrm{R}$ chart, a comparison is made using the parameter settings from the literature reviews. These parameters can be classified into three categories: $\mathrm{C}_{1}=\{\delta, \lambda, M\}, \mathrm{C}_{2}=\{\mathrm{b}, \mathrm{c}, \mathrm{g}\}$ and $\mathrm{C}_{3}=\{\mathrm{D}$, $\mathrm{F}, \mathrm{W}\}$ which involve the nature of a process, the sampling/inspection and the identification of assignable causes, respectively. The process is at a high quality level when at least two parameters of the first category are set at low levels as shown in Table 1. Similarly, the level of relevant errors from selecting/inspecting samples and identifying assignable causes is low when at least two parameters are at low levels of the second and third categories. These categories are used to classify eight tested process scenarios (Table 2).

For the computational procedures on both algorithms a computer simulation program was implemented in a Visual C\#2008 computer program. A Laptop computer ASUS with Microsoft Windows 5.1 (Build 2600.xpsp_sp2_gdr.070227-2254: Service Pack of 2) was used for computational experiments throughout.
Table 1: Process quality scenarios and their parameter levels of low $(\mathrm{L})$ and high $(\mathrm{H})$

\begin{tabular}{llll}
\hline Process quality & $\delta$ & $\lambda$ & $\mathrm{M}$ \\
\hline Low & $3.5(\mathrm{H})$ & $6.05(\mathrm{H})$ & $350(\mathrm{H})$ \\
& $3.5(\mathrm{H})$ & $6.05(\mathrm{H})$ & $50(\mathrm{~L})$ \\
& $3.5(\mathrm{H})$ & $0.05(\mathrm{~L})$ & $350(\mathrm{H})$ \\
High & $0.5(\mathrm{~L})$ & $6.05(\mathrm{H})$ & $350(\mathrm{H})$ \\
& $3.5(\mathrm{H})$ & $0.05(\mathrm{~L})$ & $50(\mathrm{~L})$ \\
& $0.5(\mathrm{~L})$ & $6.05(\mathrm{H})$ & $50(\mathrm{~L})$ \\
& $0.5(\mathrm{~L})$ & $0.05(\mathrm{~L})$ & $350(\mathrm{H})$ \\
& $0.5(\mathrm{~L})$ & $0.05(\mathrm{~L})$ & $50(\mathrm{~L})$ \\
\hline
\end{tabular}

Table 2: Summary of tested process scenarios

\begin{tabular}{|c|c|c|c|c|c|c|c|c|c|}
\hline \multicolumn{3}{|l|}{$\mathrm{C}_{1}$} & \multicolumn{3}{|l|}{$\mathrm{C}_{2}$} & \multicolumn{3}{|l|}{$\mathrm{C}_{3}$} & \multirow{2}{*}{$\begin{array}{l}\text { Process } \\
\text { scenario } \\
\left(\mathrm{C}_{1}: \mathrm{C}_{2}: \mathrm{C}_{3}\right)\end{array}$} \\
\hline$\delta$ & $\lambda$ & $\mathrm{M}$ & $\mathrm{b}$ & $\mathrm{c}$ & $\mathrm{g}$ & D & W & $\mathrm{F}$ & \\
\hline$\overline{0.5}$ & 0.05 & 50 & 1 & 0.1 & 0.0 & 0.2 & 25 & 2 & (H: L: L) \\
\hline J. & 0. & 50 & 1 & & & & & & : H) \\
\hline 0.5 & & 50 & 31 & & & & & & \\
\hline .5 & & 50 & 31 & & & & & & \\
\hline 3.5 & & 50 & 1 & & & & & & \\
\hline & & & 1 & & & & & & \\
\hline 3.5 & & 3 & 31 & & & & & & L) \\
\hline 3.5 & 6.05 & 350 & 31 & 6.1 & 0.50 & 3.25 & 325 & 320 & 8 (L: H: H) \\
\hline
\end{tabular}

Table 3: Summary of the variable choices and feasible ranges

\begin{tabular}{ll}
\hline Variables & $\begin{array}{l}\text { Feasible ranges } \\
\text { of Xbar-R chart }\end{array}$ \\
\hline $\mathrm{n}$ & $2.0-70.0$ \\
$\mathrm{~h}$ & $0.1-8.0$ \\
$\mathrm{k}_{1}$ & $0.5-7.0$ \\
$\mathrm{k}_{2}$ & $0.5-7.0$ \\
\hline
\end{tabular}

There are 100 realizations performed for each of the eight sets of tested process conditions. Each trial used the random initial design points, evenly distributed about edges, furthest from the optimum, of the safe region of operation.

Both conventional algorithms: WCMSM and LCRSOM in a context of RSM are applied to these tested process conditions for determining the performance of economic statistical designs of Xbar-R charts. Feasible ranges of both control chart variables of a sample size, a time interval, a width of an Xbar control chart and a width of an $\mathrm{R}$ control chart are selected to cover the range of values commonly found in the literature (Table 3 ). The typical response surfaces under the first process scenario with $\left(\mathrm{k}_{1}, \mathrm{k}_{2}, \gamma\right)$ fixed at $(1.5,3.5,1)$ and $(3.5,1.5,3)$ for economic statistical designs of Xbar-R chart, are shown in Fig. 4.

A process of high quality level and the low levels of relevant errors from selecting/inspecting samples and identifying assignable obviously bring the lower associated price when compared with a process of the low quality level and the high levels of relevant errors from selecting/inspecting samples and identifying assignable. 

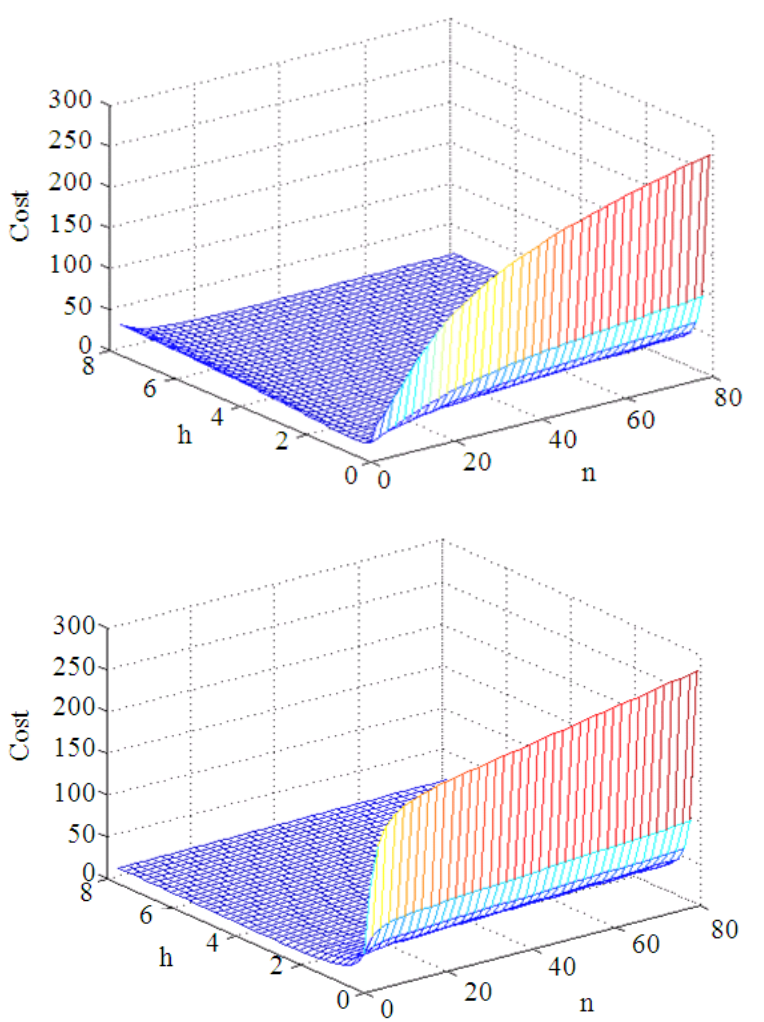

Fig. 4: Surface plot of the Xbar-R charts under a process condition: 1 with $\left(\mathrm{k}_{1}, \mathrm{k}_{2}, \gamma\right)$ at $(1.5,3.5$, $1)$ and $(3.5,1.5,3)$, respectively

Table 4: Preferable levels of Xbar-R chart variables via WCMSM

\begin{tabular}{|c|c|c|c|c|c|c|}
\hline \multirow{2}{*}{$\begin{array}{l}\text { Process } \\
\text { scenario }\end{array}$} & \multicolumn{6}{|c|}{ Xbar-R Chart Variable Levels } \\
\hline & $\gamma$ & $\mathrm{n}$ & $\mathrm{h}$ & $\mathrm{k}_{1}$ & $\mathrm{k}_{2}$ & E \\
\hline \multirow[t]{2}{*}{1} & 1 & 36 & 3.14 & 2.42 & 4.65 & 9.87 \\
\hline & 3 & 6 & 0.76 & 3.45 & 5.46 & 5.38 \\
\hline \multirow[t]{2}{*}{2} & 1 & 63 & 8.00 & 2.18 & 6.88 & 28.24 \\
\hline & 3 & 9 & 1.90 & 3.78 & 6.38 & 23.58 \\
\hline \multirow[t]{2}{*}{3} & 1 & 3 & 8.00 & 0.50 & 0.50 & 19.45 \\
\hline & 3 & 2 & 8.00 & 0.52 & 1.10 & 18.76 \\
\hline \multirow[t]{2}{*}{4} & 1 & 2 & 8.00 & 1.35 & 1.92 & 43.82 \\
\hline & 3 & 2 & 8.00 & 2.72 & 3.53 & 34.70 \\
\hline \multirow[t]{2}{*}{5} & 1 & 3 & 0.13 & 3.79 & 3.91 & 297.58 \\
\hline & 3 & 3 & 0.13 & 3.51 & 3.74 & 300.30 \\
\hline \multirow[t]{2}{*}{6} & 1 & 69 & 8.00 & 7.00 & 7.00 & 372.48 \\
\hline & 3 & 70 & 8.00 & 7.00 & 7.00 & 372.50 \\
\hline \multirow[t]{2}{*}{7} & 1 & 2 & 8.00 & 7.00 & 7.50 & 353.80 \\
\hline & 3 & 3 & 8.00 & 7.00 & 0.50 & 352.20 \\
\hline \multirow[t]{2}{*}{8} & 1 & 2 & 8.00 & 7.00 & 7.00 & 356.10 \\
\hline & 3 & 2 & 8.00 & 7.00 & 7.00 & 370.45 \\
\hline
\end{tabular}

Moreover, overall associated operating costs from process scenarios of 1-4 seem to be lower than from process scenarios of 5-8. This shows that the nature of a process seems more important than the effects from the sampling/inspection and the identification of assignable causes.

\begin{tabular}{|c|c|c|c|c|c|c|}
\hline \multirow{2}{*}{$\begin{array}{l}\text { Process } \\
\text { scenario }\end{array}$} & \multicolumn{6}{|c|}{ Xbar-R chart variable levels } \\
\hline & $\gamma$ & $\mathrm{n}$ & $\mathrm{h}$ & $\mathrm{k}_{1}$ & $\mathrm{k}_{2}$ & E \\
\hline \multirow[t]{2}{*}{$\begin{array}{l}1 \\
\end{array}$} & 1 & 55 & 1.77 & 2.13 & 7.00 & 9.58 \\
\hline & 3 & 5 & 1.19 & 3.46 & 5.29 & 4.76 \\
\hline \multirow[t]{2}{*}{2} & 1 & 3 & 4.89 & 1.42 & 3.61 & 27.05 \\
\hline & 3 & 2 & 2.44 & 1.83 & 3.66 & 23.27 \\
\hline \multirow[t]{2}{*}{3} & 1 & 2 & 2.25 & 1.68 & 3.37 & 18.57 \\
\hline & 3 & 2 & 1.67 & 3.63 & 3.54 & 17.86 \\
\hline \multirow[t]{2}{*}{4} & 1 & 4 & 1.95 & 3.73 & 3.31 & 41.26 \\
\hline & 3 & 3 & 1.83 & 3.20 & 3.33 & 34.18 \\
\hline \multirow[t]{2}{*}{5} & 1 & 3 & 0.30 & 0.50 & 0.50 & 289.36 \\
\hline & 3 & 2 & 0.28 & 1.47 & 0.50 & 298.35 \\
\hline \multirow[t]{2}{*}{6} & 1 & 4 & 0.54 & 0.50 & 1.06 & 343.58 \\
\hline & 3 & 3 & 0.55 & 1.47 & 1.06 & 353.19 \\
\hline \multirow[t]{2}{*}{7} & 1 & 2 & 0.54 & 1.47 & 0.82 & 345.70 \\
\hline & 3 & 3 & 0.54 & 1.47 & 0.82 & 345.73 \\
\hline \multirow[t]{2}{*}{8} & 1 & 2 & 0.29 & 2.85 & 0.50 & 354.49 \\
\hline & 3 & 3 & 0.29 & 1.75 & 0.50 & 358.95 \\
\hline
\end{tabular}

Number of individual measurements making up a sample or $\mathrm{n}$ is increased when a process quality level is reduced and the levels of relevant errors from selecting/inspecting samples and identifying assignable causes are increased. Interval (in hour) measured between samples or $\mathrm{h}$ and a factor used in determining the width of an $\mathrm{R}$ control chart or $\mathrm{k}_{1}$ are reduced when a process quality level decreased.

In Table 4 and 5, the results of the best of 100 realizations for economic statistical designs via WCMSM and LCRSOM, respectively, for different values of $\gamma$ and process conditions are considered. The results show that it is not significantly different to achieve the desired statistical levels of Xbar-R chart parameters when a magnitude of shift to be detected in the process dispersion $(\gamma)$ is changed from both methods. However, associated cost seems to be lower via the LCRSOM when compared with the WCMSM. A process of high quality level and the low levels of relevant errors from selecting/inspecting samples and identifying assignable obviously bring the low associated price when compared with a process of the low quality level and the high levels of relevant errors from selecting/inspecting samples and identifying assignable.

Similarly, overall associated costs from process scenarios of 1-4 seem to be lower than from process scenarios of 5-8. This shows that the nature of a process is more important than the effects from the sampling/inspection and the identification of assignable causes. Number of individual measurements making up a sample or $\mathrm{n}$ is increased when a process quality level is reduced and the levels of relevant errors from selecting/inspecting samples and identifying assignable increased. When there is an increase of $n$, levels of a process quality, errors from selecting/inspecting 
samples and identifying assignable are high, low and low, respectively. An interval (in hour) measured between samples or $h$ is reduced when a process quality level is decreased. A factor used in determining the width of an Xbar control chart or $\mathrm{k}_{1}$ has no significant result. A variable used in determining the width of an $\mathrm{R}$ control chart or $\mathrm{k}_{2}$ is reduced when the level of errors from selecting/inspecting samples is increased and the level of errors from identification of assignable causes decreased.

\section{DISCUSSION}

Some experimental runs ended prematurely because of a curved ridge surface of the cost function. The methods based on simplex designs fail to find the optimal variable levels because their solutions oscillate between one side of the ridge and the other. In summary, number of individual measurements making up a sample is increased when a process quality level is reduced and the levels of relevant errors from selecting/inspecting samples and identifying assignable are increased. An interval (in hour) measured between samples is reduced when a process quality level is decreased. Other RSM algorithms could be applied to overcome the slope of the ridge of this cost function. Operator intervention could be used to improve performance by moving the design point normal to the oscillations.

\section{CONCLUSION}

The effects of process and control chart design variables on the minimal costs, associated with a false alarm condition (before the process parameters go out of control), an out of control condition, a condition of identifying assignable cause and a sampling/inspection condition, were investigated. The control chart variables can be estimated effectively if proper experimental designs, such as factorial and simplex designs, are used to collect the data. Designs for fitting response surfaces are called response surface designs. In this study, WCMSM and LCRSOM are proposed for determining the proper levels of statistical design variables of Xbar-R control charts.

The results show that a parameter design via the LCRSOM results in a slightly lower operating cost. A process of high quality level unsurprisingly causes the low associated total costs when compared with a process of the low quality level. The process with high levels of relevant errors from selecting/inspecting samples and identifying assignable causes bring high levels of total costs. Preferable levels of control charts from both sequential methods seem to be similar.

\section{ACKNOWLEDGEMENT}

This study was supported by the National Research University Project of Thailand, Office of Higher Education Commission. The author wishes to thank the Faculty of Engineering, Thammasat University, THAILAND for the financial support.

\section{REFERENCES}

Abu-Shawiesh, M.O.A., 2008. A simple robust control chart based on MAD. J. Math. Stat., 4: 102-107. DOI: 10.3844/jmssp.2008.102.107

Baud-Lavigne, B., S. Bassetto and B. Penz, 2010. A broader view of the economic design of the X-bar chart in the semiconductor industry. Int. J. Product. Res., 48: 5843-5857. DOI: $10.1080 / 00207540903150593$

Carolan, C.A., J.F. Kros and S.E. Said, 2010. Economic design of xbar control charts with continuously variable sampling intervals. Q. Reliabil. Eng. Int., 26: 235-245. DOI: 10.1002/qre. 1050

Farshi, B., S. Gheshmi and E. Miandoabchi, 2011. Optimization of injection molding process parameters using sequential simplex algorithm. Mater. Design, 32: 414-423. DOI: 10.1016/j.matdes.2010.06.043

Ismail, Z., A. Yahya and A. Shabri, 2009. Forecasting gold prices using multiple linear regression method. Am. J. Applied Sci., 6: 1509-1514. DOI: 10.3844/ajassp.2009.1509.1514

Kamoun, A., M. Jaziri and M. Chaabouni, 2009. The use of the simplex method and its derivatives to the on-line optimization of the parameters of an injection moulding process. Chemometrics Intel. Laboratory Syst., 96: 117-122. DOI: 10.1016/j.chemolab.2008.04.010

Luangpaiboon, P., 2010. Improving an electrostatic powder coating process via signal to noise response surface. Am. J. Applied Sci., 7: 1521-1527. DOI: 10.3844/ajassp.2010.1521.1527

Matouq, M., N. Kloub and K. Inoue, 2007. The role of quality control and everyone's participation in Japan to prevent pollution during last five decades. Am. J. Applied Sci., 4: 14-18. DOI: 10.3844/ajassp.2007.14.18

Yang, S.F. and M.A. Rahim, 2009. Minimum loss design of asymmetric $\mathrm{X}$ and $\mathrm{S}$ control charts under two independent Weibull shocks. Int. J. Productivity Quality Manage., 4: 247-282. 
Yang, Y.M., C.Y. Su and W.L. Pearn, 2010. Economic design of $\bar{x}$-control charts for continuous flow process with multiple assignable causes. Int. J. Product. Econ., 128: 110-117. DOI: 10.1016/j.ijpe.2010.04.048
Zhao, Q.H., D. Urosevic, N. Mladenovic and P. Hansen, 2009. A restarted and modified simplex search for unconstrained optimization. Comput. Operat. Res., 36: 3263-3271. DOI: 10.1016/j.cor.2009.03.005 